\title{
Aus dem Funde von Tell el Amarna.
}

\author{
Von C. F. Lehmann.
}

Der Thontafelfund von Tell el Amarna ist von einer so grossen Bedeutung für unsere gesammten Anschauungen über die Geschichte und die Culturentwicklung des alten Orients, dass es angemessen erachtet wurde, gleich von Anfang an neben der Wissenschaft ${ }^{\mathrm{x}}$ ) auch die weitere Oeffentlichkeit ${ }^{2}$ ) mit den hauptsächlichsten und wichtigsten Ergebnissen derselben bekannt zu machen.

Wenn sich nun auch die, auf des Verfassers eigenen und Herrn Dr. WINCKLER's Lesungen zumeist beruhenden Angaben gegenseitig controlliren, und zudem von ägyptologischer wie von assyriologischer Seite ${ }^{\mathrm{I}}$ ) controllirt sind, so erscheint es doch wünschenswerth, der Wissenschaft von dem Inhalt und dem Charakter des Fundes einen näheren Begriff zu geben und das Wichtige nach Möglichkeit zugänglich zu machen, so dass Controlle und weitere Theilnahme an der Forschung möglich sind. Eine in Aus-

1) S. "Der Thontafelfund von Tell el Amarna" von AD. ERMAN vorgelegt und von Bemerkungen begleitet von EB. ScHRADER: Sitzungsberuchte der Königl. Prenıss. Ak. d. W. zu Berlin, 3. Mai 1888.

2) Feuilleton der ,Vossischen Zeitung" $3 / \mathrm{V} 88$; E[RMAN] ind W[INCKLER]; "Nationalzeitung" 19/V 88, Nro. 292 (H. WINCKLER); "Kölnische Zeitung" 4/VI 88, Nro. 154, (C. F. L[EHMANN]); "Hamburgischer Correspondent" 20/VI 88, Nro. I70 (C. F. LEHMANN). - Unmittelbar vor Abschluss dieses Artikels kam mir zu Gesicht: „Allgemeine Zeztung" 19/X 88, Nro. 291 (C. Bezold). Während des Druckes: BuDGE's Aufsatz PSBA X. p. 540-569 und SAYCE's Mittheilungen ebenda p. 488-525. 
sicht genommene Gesammtpublication muss naturgemäss bei dem grossen Umfange der Sammlung geraume Zeit in Anspruch'nehmen.

Im Folgenden gedenke ich daher eine Anzahl der, dem Inhalte nach wichtigeren Texte der Sammlung, die zugleich als Repräsentanten der verschiedenen, in dem Funde vertretenen Gruppen von Texten gelten können, zu veröffentlichen. Natürlich bestimmt sich die Auswahl theilweise nach dem in dieser Zeitschrift zur Verfügung stehenden Raum, sodass die umfänglichsten Texte von selbst ausgeschlossen sind.

Ich fasse zunächst einleitungsweise das Wichtigste, was über den Fund $z u$ sagen ist, wie es zum Theil bereits an anderen Stellen von Anderen wie von mir selbst veröffentlicht ist, für die Leser dieser Zeitschrift zusammen:

Die erste Kunde von dem Funde gelangte nach Berlin durch Einsendung von Gipsabgüssen mehrerer Thontafeln, aus denen ich, als sie mir zur Prüfung vorgelegt wurden, nur ersehen konnte, dass sie einen ungewöhnlichen Schrifttypus zeigten und dass einige derselben wahrscheinlich Briefe an einen König darstellten. Unter anderen bald darauf im Original eingesandten Tafeln angeblich ägyptischer Provenienz fand sich dann eine, auf

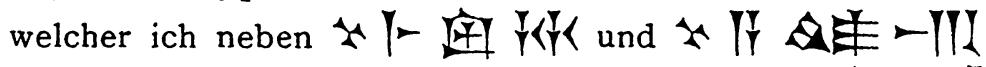
deutlich mehrmals das Vorkommen von $A$ - MV (E) constatiren konnte. Durch diese Erwähnung Aegyptens wurden die $Z$ weifel, die naturgemäss zu Anfang an der Richtigkeit der Provenienzangabe „Aegypten“ bestanden, gemindert. Unklar blieb es dagegen, welcher Zeit man die Documente zuweisen sollte: früher als an die Zeit, da Asarhaddon als König von Assyrien und Souverän Babyloniens zum ersten Mal Aegypten unterwarf, wagte auch der Kühnste nicht zu denken.

Erst die Ankunft einer grösseren Anzahl von Tafeln brachte Aufklärung über den wahren Sachverhalt: die- 
jenige Tafel, welche durch ihre Grösse und ihre schöne Schrift sofort unsere Hauptaufmerksamkeit in Anspruch nahm, erwies sich als ein Brief des Burraburias, Königs von Kara-Duniass an einen König von Aegypten, dessen verstümmelter Name sich später aus anderen Documenten zu Naphururija (d. i. Nfr-hprw-Rc ) izuerst H. Winckler) ergänzen liess, dem officiellen Namen des ägyptischen Königs, den die (xeschichte als Amcnothes') IV kennt.

Dazu stimmt die Fundstätte auf das Genaueste: Tell el Amarna liegt nämlich an derjenigen Stelle Oberägyptens, wo sich einst die Residenz befand, welche sich dieser als $\mathrm{K}$ etzerkönig berüchtigte Herrscher erbaute, um für den von ihm eingeführten Alleindienst des Sonnengottes eine Stätte zu schaffen, welche allen Einwirkungen der alten religiösen Traditionen entzogen war. Er alle in hat hier residirt; sein Nachfolger, der zur alten Vielgötterei zurückkehrte, zerstörte auch die Stadt, die den Mittelpunkt der Ketzereien gebildet hatte. Auch ein Thonsiegel Amenothes' IV hat sich gefunden.

Dass der Absender des Briefes, Burraburias, (Variante [Burn]aburiaš, in einem anderen Briefe) König von KaraDunias derselben kossäischen Dynastie zuzurechnen ist, welcher der uns als Burnaburias geläufige König angehört, daran lassen abgesehen von dem Inhalt der Briefe Namensform, Titel und alle sonstigen Umstände keine Zweifel zu.

Was die nähere Bestimmung der Persönlichkeit des Burnaburias anlangt, so erwähnt derselbe in einem seiner andern Briefe an Amenothes IV (es sind deren mindestens fünf im Berliner Museum vorhanden), dass dessen Vater also Amenothes III an Kurigalzu reichliches Gold gesandt habe. ${ }^{2}$ ) Von diesem Kurigalzu, an den der Aegypterkönig

I) So lautet der Name in griechischer Wiedergabe richtig; Amenophis, wie man diesen und die gleichnamigen Könige gewöhnlich nennt, ist die griechische Form eines andern ägyptischen Namens [Amen(em)op(h)e].

2) [A]-bu-ka (so ergänzt von Dr. WINCKLER) a-na Ku-ri-gal-zu ḩurâșa $m a{ }^{3} \cdot a-d a$ u-se-bi-i-lu (Burr. 2 Rücks. Abschn. 4). 
eine solche Sendung veranlasst, wäre schon an sich bis zum Beweise des Gegentheils anzunehmen, dass er selber König war, demnach also vor Burraburias herrschte. In einem anderen Briefe aber schreibt Burraburias geradezu an Amenothes IV: „Sintemal unsere Väter freundschaftlich zu einander gestanden haben, so wollen wir auch

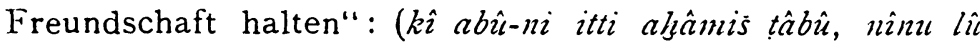
tâââul. Und ferner nennt sich nach Budge's und Bezold's Mittheilungen in einer der nach London verbrachten Tafeln Burraburias geradezu „Sohn des Kurigalzu“. Dieser Kurigalzu, Vater des Burraburias, war also König und ist von Kurigalzu sibru, der als Sohn des Burnaburias in der synchronistischen Tafel erscheint, $z u$ trennen. Letzterer ist der zweite König dieses Namens; als Kurigalzu III hat der Sohn des Ka-ara-gar-bi zu gelten. ${ }^{1}$ )

Warum aber hieraus (s. BEzold a. a. O. S. $428 \mathrm{I}$ ) folgen soll, dass der Burraburiaś-Burnaburiaśs der Tafeln von Tell el Amarna nicht mit dem aus der synchronistischen Tafel und seinen eigenen Backsteininschriften uns bekannten Burnaburias identisch ist, sehe ich nicht ein. Wenn es richtig wäre, dass jener Burnaburias der Sohn des Karaindas wäre (wie das thatsächlich EduARd MEyer ${ }^{2}$ ) und BEZoLD ${ }^{j}$ ) angeben), so könnte er natürlich nicht der Sohn Kurigalzu's I sein. Aber für diese Angabe kann ich in den Quellen keinen Anhalt finden, weder in den Inschriften des Burnaburias noch in der synchronistischen Tafel, und sehe auch, dass Tiele ${ }^{4}$ ) davon nichts sagt. Es dürfte Merer und Bezold passirt sein, was leicht genug geschehen kann,

I) Ueber die verschiedenen Kürigalzu siehe TIELE, Geschichte S. 146; WINCKLER, ZA II, S. 307 ff. und dazu TIELE, S. 6I3.

2) Geschichte des Alterthums, $\$ 27$ 1, S. 326. A uch Homares, Gesciluchte, $\mathrm{S} .428 \mathrm{f}$. hat meiner Ansicht nach keine genügende Begründung für seinen Ansatz zweier verschiedener Könige, namens Burnaburias gegeben.

3) Literatur, $\$ 30,2$, S. 58.

4) Geschichte, S. 139: "Karaindas von Babel und Asurbelniszsze von Assur . . . . . . Thre Nachfolger Burnaburijas und . . . . Bussurasur". 
dass sie einfach aus dem Umstande, dass Burnaburias in der s. g. synchronistischen Tafel auf Karaindas folgt, denselben für dessen Sohn angesehen haben. ${ }^{1}$ )

Die Briefe des Burraburiaš an den Aegypterkönig bilden die erste wichtige Gruppe dieser Texte.

Zahlreicher, umfangreicher und auch durch die Aufklärung über bisher vollständig unbekannte Verhältnisse in gewisser Weise noch interressanter sind die Berichte, welche an Nim-murija = äg. Nb-mi't-R، d. i. A menothes III, den Vorgänger und Vater Amenothes' IV gerichtet sind, von dem Könige Duschratta von Mitanni. Dieselben handeln vornehmlich von der Vermählung der Tochter des Duschratta mit dem Aegypterkönige und von der letzterer zugedachten Mitgift, deren Bestandtheile in langer Liste aufgezählt werden, etc. Die Tafeln - schon äusserlich an einer besonderen rothen Farbe des Thones kenntlich sind mit schöner babylonischer, leider vielfach jetzt durch Verwitterung undeutlicher, Keilschrift in babylonischer Sprache beschrieben. Dieser Gruppe gehören die grössten Thontafeln an, die unseres Wissens überhaupt bis jetzt gefunden worden sind: eine derselben erreicht eine Höhe von nahezu $1 / 2$ Meter.

Einer dieser Briefe des Duschratta trägt àm Rande mit ägyptisch-hieratischer Schrift in schwarzer Farbe aufgemalt einen ägyptischen Vermerk, wie wir solchen auf diesen Tafeln mehrfach begegnen. ERMAN übersetzt denselben folgendermassen: „[Jahr] $2^{2}$ ), erster Wintermonat, Tag...., als der Hof sich in der südlichen Residenz, in der Burg Ka-em-echut aufhielt. Duplicat(?) des

I) Meine und wohl auch ED. Meyer's Angaben basirten auf der Lit. S. I3, § ro, 4 erwähnten Collation und Ergänzung DelıTzsch's; vgl. besonders Kossäer, S. 7, Note. [Dagegen freilich jetzt die „Collation und Copie“" WINCKLER's; s. SCHRADER's Bibliothek I, S. 194 ff.]. - Bezoll.

2) Dic 2 ist sicher, ob aber nicht vorher das hieratische Zeichen für 30 oder für 40 gestanden hat, muss unentschieden bleiben (STrindoRFF). 
Naharina-Briefes, welchen der Bote Petreza und der Bote ..... gebracht haben".

In der Vorstellung des Schreibers dieser Notiz fallen also das Land Mitanni und das längst bekannte Land Vaharina zusammen. Daraus könnte man schliessen, dass beide Länder völlig identisch wären, und class Mitanni der einheimische Name des Landes Naharina sei, welches den Aegypterkönigen so viel Schwierigkeiten bereitete. Es ist aber auch denkbar, dass die Notiz eine ungenaue Angabe enthält, dass Naharina im Sinne des Schreibers nur ein allgemeiner Name für die am mittleren Euphrat gelegenen Staaten war, und dass also entweder Mitanni der Name eines selbständigen, vielleicht hervorragenden Theils dieser Staatengruppe, oder aber dem Lande Naharina benachbart war. Gegen die Annahme völliger Identität spricht besonders der Umstand, dass, wie von verschiedenen Seiten ${ }^{1}$ ) sogleich bemerkt wurde, ein in Asien gelegenes Land Mtn in den Inschriften der Könige mehrfach genannt wird.

Nach gütiger Mittheilung ERMAN's kommt dasselbe z. B. vor in der vom Könige Thutmosis III stammenden Stele von Karnak, die einen poetischen Bericht von dessen Thaten enthält - in einer Aufzählung der von ihm besiegten Völkerschaften. Es werden da genannt: 1) die Grossen von Phönikien; 2) die, welche im Lande Setet (= Asien) sind, und die Amu von Syrien; 3) das östliche Land und die in den Gauen des Gotteslandes; 4) das Westland Kefti und Asebi; 5) die, welche in ihren Nebu(?) sind, die Länder von $M t n$; 6) die, welche auf den Inseln sind; 7) das Land Telicnu und die Inseln von Utena.

Aus dieser ziemlich wirren Aufzählung, die auf Beachtung der geographischen Lage keinen Anspruch erhebt, kann man höchstens schliessen, dass mit Mtn ein vorderasiatisches Land gemeint ist, das nicht allzuferne von Kilikien zu suchen wäre.

1) Besonders auch von BRUGSCH in der Vossischen Leitung, Nr. 283. Sonntags-Beilage 17 /VI 88 . 
Eine Städteliste Ramıses' III nennt dagegen hinter Karkemisch (Gargamiš) ein Man. Damit stimmt, dass das am oberen rechten Euphratufer gelegene') Land ${ }^{2}$ ) IY $E Y ー \sim$ (Hanigalbat?) in den Briefen des Duschratta mit Mitanni zusammengenannt wird und also ebenfalls jenem Lande benachbart sein muss. Schliesslich erzählt Tiglatpileser I (Col. VI, 59), dass er in der Wüste im Lande Mitâni und in der Stadt Araziḳi, sa pân (mât) Hatti, Jagd auf Stiere gemacht habe. Araziki ,ist aber fragelos das Eragiza des Ptolemäus und $p$ in des Talmud", ist demnach, wenn es für den von Osten kommenden Assyrerkönig vor dem Lande der Chetiter liegt, östlich von diesem und von seiner Hauptstadt Gargamiš zu suchen. Demnach darf man das Land Mitanni mit Schrader ${ }^{3}$ ) localisiren auf das Gebiet zwischen den Flüssen Euphrat und Balich = Belias. woraus sich der Name Naharina $=$ aram. 'Zweistromland" erklärt. Ob ein Theil dieses Gebietes speciell den Namen Mitanni führte, und welcher, bleibt. noch aufzuhellen.

Die an Amenothes III adressirten Briefe können nicht ursprünglich an dem jetzigen Fundort auf bewahrt gewesen sein; sie müssen aus Theben, der Residenz der Aegypterkönige, wohin sie zunächst gelangt sein werden, von Amenothes IV als Theile des Archivs seines Vorgängers bei der Verlegung seiner Residenz mit fortgenommen worden sein. Vermuthlich dienten Alabastertäfelchen als Verschlusssiegel der Behältnisse, in welchen die Documente aufbewahrt wurden. Ein Theil der Thontafeln wurde in Tell el Amarna noch in einem Thongefäss gefunden, sowie eine Anzahl Alabastertäfelchen, welche die beiden Namen AmenothesNimmurija in ägyptischen Hieroglyphen tragen. Der erstere Name zeigt auf allen Exemplaren deutliche Spuren eines

1) S. SCHRADER, KGF I5 I ff.

1) Vgl. WinCKLer bei ERMAN-Schrader, Sitzungsber. a. a. O. S. 5 .

3) SCHKADER KGF $22 S$. 
Tilgungsversuchs: Amenothes IV, als Verfolger der althergebrachten Religion, änderte seinen eigenen Namen um, weil er den Gottesnamen Ammon enthielt und tilgte diesen Gottesnamen, wo er ihn antraf - so auch im Namen seines Vorgängers. - Hervorzuheben sind ferner Briefe des Königs von Alašija an den Aegypterkönig.

Von den übrigen Tafeln, soweit ich sie nach ihrem Inhalt bis jetzt $z u$ überblicken vermag, sind diejenigen die interessantesten und in grösster Zahl vertretenen, die aus Syrien und vorı der phönikischen Küste nach Aegypt gesandt sind. Es fanden sich eine Anzahl Berichte von Präfecten syrischer unter ägyptischer Oberhoheit stehender Städte an den Aegypterkönig in babylonischer Sprache. Auf ihnen erscheinen u. A. die folgenden Städte:

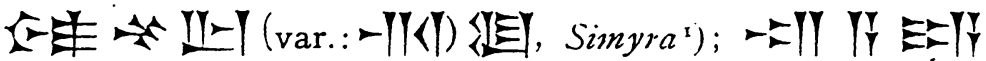
出 auf einer der oben erwähnten in Gypsabguss zuerst nach

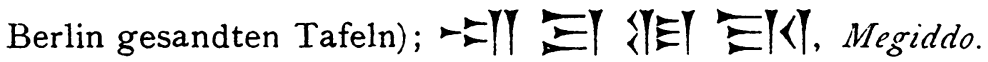
Auch $G u-u b-l i$ (= Byblos) und As-ka-lu-na (= Askalon) kommen vor. $\left.{ }^{2}\right)$

Unter diesen Briefen aus Syrien finden sich nun auch solche, die nicht direct an den König gesandt sind, sondern als Adressaten hohe Würdenträger am Hofe oder im Dienste des Aegypterkönigs nennen. Ein solcher ist die von einem Azinu aus dem nördlichen Syrien an $Y$ Y $H \hat{a}-i$, einen öfters genannten ägyptischen Beamten, gesandte Botschaft, welche Hilferufe gegen den Angriff drohenden Chetiterkönig enthält und als bereits von diesem erobert die Stadt $D u-n i$-ib (Z. 25), d. i. äg. Tvnp ${ }^{3}$ ) nennt. Dieselbe berichtet in diesem Zusammenhange, dass

I) Vgl. Delízsch, Par. S. 28 I f.

2) Schrader, a. a. O., S. 6.

3) ERMan, a. a. O., S. 3. 
der König von Kurhaše, einem Lande, das sich verschiedentlich in diesen Inschriften genannt findet, in das Land $=1 /$ KE, Martu, geflohen sei. Von demselben Absender sind noch fernere Botschaften vorhanden, die ebenfalls von grosser Bedrängnis durch die Chetiter reden. Eine derselben ist gerichtet an $D u-d u$, einen ägyptischen Würdenträger, der vielleicht mit dem Tu-tu identisch ist, dessen Grab bei Tell el Amarna man seit längerer Zeit kennt.

Ganz isolirt steht eine Tafel, welche aus mehreren Bruchstücken im Berliner Museum fast vollständig wieder zusammengesetzt ist - ein auch seinem Inhalte nach für uns sehr wichtiger mythologischer Text, der von einem ägyptischen Schreiber zu einer Leseübung benützt worden ist, die er natürlich an einem der officiellen Staatsdocumente nicht in dieser Weise hätte vornehmen dürfen: er hat mit Punkten in rother und schwarzer Farbe die Wort. enden bezeichnet, wie ich dies in der Textausgabe dieser Tafel nach Möglichkeit veranschaulichen werde.

Diese gedrängte, die Urkunden ihrer Herkunft und ihrem Inhalte nach nicht entfernt erschöpfende Uebersicht wird genügen, um erkennen zu lassen, in welchen Richtungen sich uns hauptsächlich aus dem Funde von Tell el Amarna neue Aufschlüsse und Erweiterung unserer Kenntnisse ergeben, und auf welche Punkte wir somit bei der Besprechung der Texte das Hauptaugenmerk werden zu richten haben.

Was zunächst die Schrift anlangt, so war die Ausbildung der babylonischen Cursivschrift - die Benennung neubabylonisch wird man wohl jetzt als wenig passend nach Möglichkeit vermeiden - schon in sehr alter Zeit weit gediehen; dieselbe ist uns bereits aus den Contracttafeln aus den Zeiten der sog. ersten babylonischen Dynastie, wie sie im Britischen Museum und in besonders reicher Zahl im Berliner Museum vorhanden sind, bekannt. 
Die theilweise mit grosser Feinheit geschriebenen Briefe des Burraburias, die mythologische Tafel und der Brief des Duschratta von Mitanni, unterscheiden sich nicht wesentlich von der babylonischen Cursivschrift, wie wir sie aus den $\mathrm{Cy}$ -

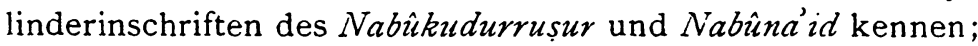
allerdings zeigen dieselben, und das gilt namentlich von den aus Mitanni stammenden Briefen, hin und wieder Formen, die mehr an den Schriftstyl erinnern, den wir als den assyrischen zu bezeichnen gewöhnt sind. - Natürlich ist die Identität mit der Cursivschrift cum grano salis zu verstehen, denn gewisse Zeichenformen weisen deutlich auf die nähere Verwandtschaft mit altbabylonischen Zeichen zurück. Dies gilt namentlich von den Zeichen für šarru und amelu, die stetig die drei senkrechten Keile in dem untern wagrechten Balken zeigen, welche für die alte Form dieser Zeichen unerlässlich sind. Hierher gehört auch die strenge Unterscheidung von Zeichen, die in späterer Zeit vielfach zusammengeflossen sind, oder leicht verwechselt werden; so namentlich bei Burraburias ba ( $\langle\mathbf{Y}\rangle$ ) und ( 1 u. 2 ) ma (E). Die Thatsache, dass babylonis che Sprache und Schrift im zweiten vorchristlichen Jahrtausend in ganz Vorderasien bis nach Aegypten als Verständigungsmittel im internationalen Verkehr im Gebrauch sind, spiegelt sich in einer gewissen Mannigfaltigkeit in der Form der Zeichen und in einzelnen Abweichungen von der uns bekannten Norm der babylonischen Cursivschrift wieder. Sind die Briefe des Königs Duschratta von Mitanni, abgesehen natürlich von ihrem Erhaltungszustande, für den des Neubabylonischen Kundigen ohne Schwierigkeit zu lesen, so macht bei den aus syrischen Städten und anderen vorderasiatischen Gegenden stammenden Briefen die Schrift vielfach zunächst einen ausserordentlich fremdartigen Findruck. Nach einiger Zeit der Uebung wird man jedoch finden, dass derselbe vielfach mehr auf Rechnung von Eigenthümlichkeiten im Ductus als von wirklichen Ver- 
schiedenheiten in der Form zu setzen ist, und man wird jedenfalls zugeben müssen, dass wir es hier so ziemlich überall mit Varietäten der bisher sog. neubabylonischen Schrift $z u$ thun haben. ${ }^{\mathrm{i}}$ )

Zur Beurtheilung der babylonischen Sprache dieser Denkmäler sind natürlich zunächst die direct aus Babylonien stammenden 'Tafeln, d. h. also die Briefe des Burraburias und die mythologische Tafel, dann erst die von der einheimischen Sprache nicht immer unbeeinflussten Briefe anderer Absender heranzuziehen. Der Umstand, dass die ersteren fast durchweg phonetisch geschrieben sind, der sich wohl aus dem Bestreben erklärt, für den ausländischen Leser, für welchen die Briefe bestimmt waren, die Schwierigkeiten der babylonischen Schrift nach Möglichkeit $z u$ mildern, befähigt uns ein einigermassen deutliches Bild von der damaligen Gestalt der Sprache zu gewinnen. Dieselbe zeigt im $\mathrm{W}$ esentlichen alle Merkmale der uns aus Documenten mittel- und neubabylonischer Zeit bekannten Sprache.

Der Lautbestand zeigt, dass die Wandlungen bereits vollzogen sind, die uns ein Vergleich der späteren Documente mit den Proben, welche uns aus Hammurabi's Zeit erhalten sind, erkennen lässt. Die Versuche, gewisse, der semitischen Sprache eigenthümliche Laute, wie namentlich das, (vgl. 2. B. $F-$ If murabi ZA II, S. 75), $y$, ', 7 mit dem vorhandenen unzureichenden Material auszudrücken, Versuche, die für den, der sehen will, deutlich zeigen, dass das Babylonische in seiner Entwicklung eine Stufe hinter sich gelassen hat, in der es die Eigenthümlichkeiten der übrigen semitischen Sprachen in weit höherem Masse theilte, als es später den Anschein hat, - diese Versuche sind in Wegfall gekommen oder treten nicht mehr so. deutlich hervor. Dagegen finden sich gewisse Abweichungen, die wir als

1) Vgl. Schrader, Sitzungsberichte a. a. O. sub 2, S. 5. 
charakteristisch für den babylonischen Dialect im Gegensatz zum assyrischen seit langem kennen, wieder, so z. B. die Vertretung der Emphatica durch die entsprechende Sonans, namentlich häufig $g$ für $k$. Wenn wir derartige Eigenthümlichkeiten regelmässig in den baylonischen Documenten aller Zeiten, von Hammurabi ( $z$ für $s$ ) bis $\mathrm{Ne}-$ bukadnezar und Nabonid finden, so glaube ich ganz entschieden, dass dieselben eine sprachliche Eigenthümlichkeit wiederspiegeln, und kann mich nicht mit HaupT und Flemming ${ }^{i}$ ) dazu verstehen, darin lediglich eine willkürlich graphische Eigenart zu sehen, die in ihrer mit grosser Regelmässigkeit gepaarten Willkürlichkeit unverständlich wäre, zumal da doch dieselben Erscheinungen in andern semitischen Sprachen vorkommen, arabisch ت z. B. im Aegyptischen $z \mathrm{u} F(g)$ wird, im syrischen Dialect gar sich zu 1 verflüchtigt. Allerdings sind es Erscheinungen, die in den einzelnen semitischen Sprachen erst auftreten, wenn dieselben auf einer ziemlich vorgeschrittenen Stufe der Entwicklung und der lautlichen Zersetzung stehen. Dass aber die vorliegenden Texte $z u$ allen andern ein gewichtiges Zeugnis dafür hinzufügen, dass wir es in der assyrisch-babylonischen Sprache mit einer lautlich weit vorgeschrittenen und in der Zersetzung begriffenen Sprache $z u$ thun haben, das steht mir so fest, wie ich andererseit davon überzeugt bin, dass sie von denen, die im AssyrischBabylonischen noch immer das Sanskrit der semitischen Sprachen sehen (und aus dem Mangel eines Ausdrucks für gewisse Laute der Keilschrift auf ein Fehlen derselben in der ursemitischen Sprache schliessen), später zum Beweise für ihre gegentheilige Behauptung werden aufgeführt werden.

Ausser den bereits aus der Vergleichung des Lautbestandes der Texte des Burraburiaš und des Hammurabi

1) Die grosse Steinplatteninschrift Nebucadnezar's, S. 27. [S. aber auch schon meine Diss., Darizusins., S. 25 und Nn. 2, 3. - Berold]. 
gezogenen directen Beweisen gegen eine solche verkehrte Auffassung liefert uns der Fund von Tell el Amarna noch ein gewichtiges indirectes Zeugnis durch seine Aufschlüsse über die reiche Entwicklung der babylonischen Cultur in einer frühen Zeit. Denn es kann bekanntlich als eine Regel gelten, dass $z$ wischen der sprachlichen Entwicklung und der Geschichte. eines Volkes gewisse Wechselbeziehungen bestehen. Das Volk, dessen historisches Leben früh beginnt, das früher zur Ausübung seiner Culturmission gelangt, von dem kann man auch erwarten, dass seine sprachliche Entwicklung frühtr in Fluss gerät, die Sprache sich dem practischen Bedürfnisse anbequemt, dass ihr Wortschatz bedeutungsreicher, dagegen zumeist ihr Lautbestand und ihre Formenbildung vereinfacht werden ${ }^{\mathrm{I}}$ ).

Deshalb tritt uns die Sprache der Araber, die von allen semitischen Völkern in die Geschichte am Spätesten eingegriffen haben und deren Entwicklung sich sozusagen vor unseren Augen abspielt, namentlich was das Lautsystem anlangt, in der ursprünglichsten Gestalt entgegen, und so lange es eine Sprachwissenschaft giebt, so lange wird das Arabische, aus so später Zeit wir es erkennen, als wichtigste $Q$ uelle für den Lautbestand des Semitischen überhaupt angesehen werden. Und mit aller Entschiedenheit muss dagegen Verwahrung eingelegt werden, wenn z. B. aus dem - nominellen (s. o. S. 382) - „Fehlen“ eines, $\varsigma$ in der babylonisch-assyrischen Schrift der Schluss gezogen wird, diese Laute, welche in den sämmtlichen übrigen semitischen Sprachen an den entsprechenden Stellen

I) Aus dieser an sich richtigen Beobachtung ist, wie EDUARD MEYER (Geschichte des Alterthums. § I Anm., S. 2) mit Recht hervorhebt, die irrige und irreführende Ansicht SCHLEICHER's entstanden, dass Sprachbildung und Geschichte im Leben des menschlichen Geistes sich ablösende Processe seien. Nur so viel ist richtig, dass mit der geschichtlichen Entwicklung vielfach eine lautliche Zersetzung Hand in Hand geht, die ein neues Moment in der Bildung und Entwicklung der betr. Sprache, aber gewiss kein Aufhören dieser Entwicklung bezeichnet. 
erscheinen, hätten sich erst secundär entwickelt, eine Anschauung, die seit I 884 von $\mathrm{H}_{\mathrm{AUPT}}{ }^{1}$ ) $z$ war $z$ weifelnd, aber mit wachsender Neigung zu grösser Bestimmtheit vorgetragen wird. Darnach wäre ı̀mu die ursprüngliche Form, das $\sqrt{ }$ in etc. secundär entwickelt, wäre alâdu älter als 4 , ילד, $u_{\text {ي und }} T$, hätten das Suffix der ersten Person Sing. am Nomen und das Verbalpräfix des Imperfects ursprünglich kein ى, wäre also iškun alter als 'يَنَ')

Gerade die langsame Veränderung des Arabischen kann uns zeigen, eine wie lange Entwicklung wir schon anzunehmen haben, wenn wir die babylonische Sprache aus der Zeit des Burraburiaš nach den geschriebenen Monumenten beurtheilen - gesprochene und geschriebene Sprache absolut $z u$ identificiren ist nirgends weniger am Platze als im Babylonisch-Assyrischen, einer semitischen Sprache mit gänzlich unsemitischer Schrift - ; ist doch schon die Periode von Narâm-Sin bis Burraburiaš, wenn wir den bekannten Angaben Nabuna'ids Glauben schenken, länger als die Zeit, die von der Begründung des Islam bis auf unsere Tage verstrichen ist. Das Babylonische. aus Burraburias' Zeit unterscheidet sich gewiss ebenso sehr von der ursprünglichen Sprache zu Beginn des selbständigen Volkslebens der Babylonier, wie das Hebräische in der Scheinsprache, die uns die Masorethen auftischen von dem Hebräischen, wie es gesprochen wurde zur Zeit der Abfassung des Deborah-Liedes, oder wie das heute in einer ägyptischen oder syrischen Stadt gesprochene Ara-

1) S. 2. B. Fohns Hopkins Uinizersity Circulars 1884, No. 29, p. 51, ZA II, $287 \mathrm{f}$. Anm. und Prolegomena to a Comparatiz'e Assyrian Grammar, pp. LIII, LXI.

2) Eine ausführliche Würdigung und Widerlegung der scheinbar für diese Ansicht sprechenden Beweise und Analogien muss ich mir für eine andere Gelegenheit aufbehalten. Im Allgemeinen s. NöLDEKE, Die semitischen Sprachen, S. 4. 5. 4 I etc. 
bisch von der Sprache der Beduinen und selbst der Städter zu Muhammed's Zeit. ${ }^{\mathrm{x}}$ )

Ein zweiter Punkt, bei dem es gerathen erscheint, einer zu erwartenden irrthümlichen Ausbeutung der durch den Fund von Tell el Amarna dargebotenen neuen Data und Gesichtspunkte von vornherein einen Riegel vorzuschieben, ist die Frage nach der Existenz der sumerischakkadischen Sprache.

Die Briefe des Burraburias und die mythologische Tafel sind in reinem semitischen Babylonisch abgefasst. Bekanntlich haben wir aber von den Kossäerkönigen, von burraburiaś wie von seinen Vorgängern und Nachfolgern auf dem babylonischen Throne, Backsteinlegenden in protobabylonischer Redaction, von denen man bereits behauptet hat, dass sie nach Absicht ihrer Verfasser lediglich als ideographisch geschriebenes Semitisch aufzufassen seien²), eine Frage, über die sich streiten lässt, wenn wir uns auch vorläufig zu der gegentheiligen Ansicht bekennen müssen. Nun da die Briefe des Burraburiaš uns das schönste semitische Babylonisch vor Augen führen, müssen wir darauf gefasst sein, dass die Leugner der Existenz einer protobabylonischen, sumerisch-akkadischen Sprache sich jubelnd auf diese Thatsache berufen werden, um mit erneuter Bestimmtheit zu behaupten: „Der Akkadismus ist todt!“ „Der babylonische Kossäerkönig schreibt an den König von Aegypten in semitisch-babylonischer Sprache, folglich sind seine Backsteinlegenden babylonisch, folglich hat es nie. eine andere als die semitische Sprache in Babylonien gegeben!'” Die Erwägung, dass -die babylonische Sprache

I) Die wichtige Frage, in wie weit Sprache und Schrift sich decken, in wie weit die Unvollkommenheiten der letzteren gewisse Neigungen zum lautlichen Schwunde in der Sprache zunächst der Schriftkundigen zu schnellerer Entwicklung gebracht haben, muss hier undiscutirt bleiben.

2) Pognon; s. Halévy, Revue critigue, r884, No 29, p. 47 , n. 1 ; ZIMMERN, Busspsalme'n, S. 4 . 
für die Herrscher aus kossäischem Geschlecht eigentlich auch eine Fremdsprache war, die sie so gut anwendeten, wie z. B. heutzutage ein Deutscher auf dem griechischen Throne sich des Neugriechischen im officiellen Verkehr $z u$ bedienen hätte, wird die Antiakkadisten wohl kaum dazu bewegen, die Möglichkeit der Existenz einer nichtsemitischen Sprache in Babylonien zuzugeben; hat doch

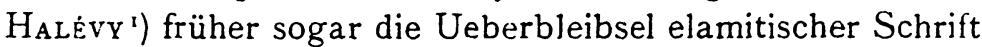
ebenfalls für graphische Variationen der einen semitischbabylonischen Geheimschrift erklärt, - die nur noch von einigen gänzlich Verstockten als Ausdruck einer Sprache angesehen wird. Dagegen wird es vielleicht dazu dienen, die Freude der Antiakkadisten über diesen scheinbar neuen Beweis für ihre Ansicht in etwas zu dämpfen, wenn wir ihnen mittheilen, dass keiner von den Vertretern des „Akkadismus" die Behauptung aufstellen wird, es habe nach den Königen der sog. ersten babylonischen Dynastie ein babylonischer Herrscher sich des Protobabylonischen als des Ausdrucks einer noch lebenden gesprochenen Sprache bedienen können. Nach dem Siege, welcher Hanmurabi die Herrschaft über ganz Babyloniens verschaffte, wird man die Fortexistenz. des sumerischakkadischen Elementes in massgebenden Schichten der babylonischen Bevölkerung nicht lange mehr annehmen dürfen, - wie lange vorher auch im südlicheren Theile Babyloniens das semitische Element die Oberhand gewann, entzieht sich einstweilen unserer Beurtheilung. Herrscht somit darin zwischen Akkadisten und Antiakkadisten die schönste Einigkeit ${ }^{2}$ ), so fällt auch für die Gegner die Mög-

1) Aperçu grammatical de l'allographie ass.ry-babylonienne. Leydener. Congressacten II S. 567 , sub 3 .

2) Unserer Ansicht nach wird überhaupt der ganzen, jetzt so sehr in Blüthe stehenden antiakkadischen Bewegung durch klare und deutliche Formulirung einer Anzahl von - eigentlich selbstverständlichen Zugeständnissen der Boden entzogen. Näheres siehe in meinem Šamassumukin, Cap. IV, und vgl. ZimMERN, Babylonische Busspsalmen, S. $5 \mathrm{ff}$. 
lichkeit fort, den Fund von Tell el Amarna gegen die Existenz der sumerisch-akkadischen Sprache auszuspielen. Der Schwerpunkt der Frage liegt in der Zeit lange vor Burraburias, lange vor Hammurabi. Auf diesen Kampfplatz laden wir unsere Gegner.

Ueber Einzelheiten der Grammatik bringen die Commentare Näheres. Wir verweisen hier nur auf die sehr häufige Verwendung der s. g. Permansivformen vom einfachen Verbalstamm als einer lebendigen Form der gewöhnlichen (nicht poetischen) Schriftsprache.

Der Gewinn, den die Geschichte des alten Orients aus diesem Archiv eines um die Mitte(?) des fünfzehnten Jahrhunderts v. Chr. herrschenden Aegypterkönigs ziehen wird, kann nicht hoch genug angeschlagen und zur Zeit kaum vollständig ermessen werden. Was zunächst die genannte annähernde chronologische Fixirung der beiden Herrscher Burraburias und Amenothes I $V$ anlangt, so überlasse ich die nähere Discussion und Begründung dieses Ansatzes denjenigen, die sich zur Zeit speciell mit chronologischen Studien befassen. Dass dieser urkundliche Beweis für die Gleichzeitigkeit beider Herrscher für die bisher ausserordentlich schwankende Chronologie sowohl Aegyptens wie Babyloniens von grossem Werthe ist, ist ohne Weiteres klar.

Dass Aegypten und Babylonien in so früher Zeit in geregeltem Verkehr standen, ist uns neu; dass neben diesem „commercium" auch ein „connubium" der Herrscherfamilien erscheint, und dass die Familienbeziehungen zur Stützung und Erhaltung politischer Beziehungen gesucht und benutzt wurden, ist unter diesen Umständen nicht überraschend. Verwunderlich aber wäre die grosse Anzahl der Bündnisse, das einmüthige Zusammenstehen so vieler verschiedener Staaten, - Aegypten ist mit Mitanni durch die Verheirathung des Amenothes III mit einer Tochter des Königs Duschratta verknüpft; Burraburias scheint eine ägyptische Prinzessin zur Frau zu haben 
und schlägt die Erneuerung von älteren Bündnissen vor; und aus der synchronistischen Tafel $^{\mathrm{x}}$; wissen wir, dass gleichzeitig $z$ wischen der babylonischen Kossäerdynastie und den Herrschern des damals aus kleinen Anfängen emporkommenden Assyrien verschiedentlich die engsten Familienbeziehungen geknüpft und zugleich Bündnisse geschlossen wurden ${ }^{2}$ ) - , diese grosse Friedfertigkeit wäre verwunderlich, wenn man nicht wenigstens bei einem Theil der Staaten, die solche Friedensliebe an den Tag legen, vermuthen dürfte, dass sie sich von einer Seite Ruhe verschafften und womöglich Unterstützung zusịchern liessen, um desto energischer ihre Kräfte auf einen andern bedrohten Punkt zu concentriren.

Dies dürfte direct für Aegypten und für Mitanni zutreffen.

Die Tafeln führen uns in die Zeit, wo die Chetiter (s. o. S. 379) sich zu regen beginnen und den Grund zu jener Machtstellung legen, in der sie namentlich den Aegyptern so viel zu schaffen gemacht haben, und die zu brechen, oder wenigstens von der Offensive abzubringen erst den Herrschern der folgenden (19.) Dynastie gelang. Die aus Syrien an ägyptische Beamte gesandten Hilferufe zeigen, dass die Chetiter schon jetzt in die ägyptische Machtsphäre eingreifen; und dass Mitanni von solcher Bewegung in irgend einer Weise in Mitleidenschaft gezogen werden musste, ist bei seiner oben geschilderten Lage beinah selbstverständlich.

Es steht $z u$ hoffen, dass bei weiteren Studien der Fund von Tell el Amarna auch über die Chetiter ${ }^{3}$ ), ihre Geschichte, ihre Nationalität, ihre Sprache Licht ver-

I) II R 65, No 1, III R 4, No 3 .

2) Ueber directe Beziehuugen zwischen Assyrien und Aegypten in dieser Zeit, wie sie aus Theilen des Fundes von Tell. el Amarna, die sich nicht in Berlin befinden, hervorgehen, werden wohl demnächst von anderer Seite Mittheilungen gemacht werden.

3) Näheres von anderer Seite. 
breiten wird, so dass wenigstens die Möglichkeit angebahnt wird, sie als einen Volkskörper in ihrem Werden, ihren Eigenthümlichkeiten, ihrer Sprache und ihren Geschicken zu betrachten, statt dass sie uns, wie doch jetzt, nur theils als Störenfriede erscheinen, an denen Aegypter, Assyrer und syrische Völkerschaften ihre Tapferkeit und die Schärfe ihrer Waffen erproben, theils als wichtige Faktoren in der Vermittlung und Verschmelzung auswärtiger Einflüsse und als Träger derselben.

Um die Bedeutung des Fundes in der soeben erwähnten culturgeschichtlichen Richtung zu würdigen, würde es schon an sich genügen, das bis dahin völlig unbekannte Factum hervorzuheben, dass die babylonische Sprache und Schrift im zweiten vorchristlichen Jahrtausend im internationalen Verkehr, und zwar nicht blos zwischen Babylonien und seinen Nachbarstaaten, sondern auch zwischen Staaten, die mit Babylonien nichts gemein haben. und selbst im inneren Verkehr des ägyptischen Reiches - in so ausgedehnter Weise in Aufnahme kommen konnte, eine Verwendung, die man sich übrigens ohne eine weitgehende Beschäftigung babylonischer Schriftgelehrter als Lehrer, Dolmetscher und Beamte ausserhalb Babyloniens nicht wird erklären können. Dies wird bestätigt durch die Erwähnung des Dolmetschers targumannu, der dem Ueberbringer eines der Briefe des Duschratta von Mitanni beigesellt wurde, wie in dem Documente selbst erwähnt wird ${ }^{1}$ ).

Aber die Tafeln von Tell el Amarna geben uns directe Aufschlüsse, die die Vorstellung, welche wir uns auf Grund der Verbreitung der babylonischen Schrift über die culturhistorische Bedeutung Babyloniens gebildet haben würden, bestätigen und erläutern.

Neben den politischen Beziehungen der Herrscher her und vermuthlich zeitlich ihnen voraus müssen als wichtigste Träger und Vermittler der Cultureinflüsse die $\mathrm{H}$ andelsbe-

1) IVINCKLER bei SCHRADER a. a. Q. S. 7. 
ziehungen gegangen sein. Ein ungefähres Bild von A ngebot und Nachfrage lässt sich aus den Geschenken entnehmen, die sich die Herrscher senden und aus den Gegenständen, die die Mitgift der Tochter des Duschratta ausmachen. Denn es sind natürlich vornehmlich Producte des eigenen Landes, die in der Fremde, in die sie gesandt werden, einen hohen Werth haben, weil sie dort ganz fehlen oder selten und schwer erreichbar sind. Von besonderem Interesse ist beispielsweise, dass Burraburias an Amenothes IV als Friedensgeschenk fünf $\mathrm{Ge}$ spanne von Rossen sendet. Bekanntermassen ist das Pferd aus Asien nach Aegypten eingeführt ${ }^{x}$ ) und wird in clen ägyptischen Documenten und auf den Denkmälern erst zu Beginn der achtzehnten Dynastie (der Thutmosis IV = Mimmurija, Amenothes III und IV angehören) und zwar immer als ein seltner und hochgeschätzter Artikel erwähnt.

Die in Lydien ${ }^{2}$ ) erfolgte Erfindung des gemünzten und geprägten Geldes, d. h. der Ausbringung von Stücken (edlen) Metalles, deren bestimmtes Gewicht und Feingehalt durch die Prägung königlicher-oder staatlicherseits gewährleistet wurde, ist nur die Folge und letzte Entwicklung eines in altorientalischen Verkehr lange vorher geübten Brauches ${ }^{3}$ ). Namentlich die Edelmetalle Gold und Silber liefen in grösseren und kleineren Stücken von sorgfältig bestimmtem Gewicht um; theils um eine bemerkbare Verringerung auszuschliessen, theils zur bequemeren Handhabung gab man ihnen eine regelmässige Form (Ringe, Kugeln, Barren); und Gewicht und Reinheit wurden durch Aufdrückung eines Stempels seitens der Ausgabestelle garantirt.

1) Darüber zuletzt ViRchow in einer Sitzung der Berliner anthropo. logischen Gesellschaft.

2) Herodot, I, 94 ; Pollux IX, 83.

3) S. Brandis, Das Münz-, Mass- and Geaichtsaicsen in Vorderasien bis auf Alexander din Grossen.

Zeitschr. f. Assyriologie, 111 . 26 
Wie gesagt, das Bestehen dieses Brauches in sehr alter Zeit war vermuthet und überliefert. Ein so altes directes Zeugniss für denselben, wie uns der Brief des Burraburias liefert, der von dem Aegypterkönige als Gegengabe für sein Friedensgeschenk Gold erbittet ${ }^{\mathrm{I}}$ ) und - wie es scheint, unter Hinweis auf eine frühere Unterlassung in dieser Richtung - ausdrücklich hinzufügt, dass sein königlicher Bruder es „prüfen“ und ,stempeln" lassen möge (anders sind die Ausdrücke hurașu .... abûa limur-ma liknuk doch kaum zu verstehen), war bisher meines Wissens nicht bekannt.

Wie die babylonische Schrift den internationalen Gedankenaustausch in jener Zeit regelte, so normirte babylonisches Gewicht die Metalle als Werthmesser des internationalen Waarenverkehrs; das System der babylonischen Mine, das im Laufe der Zeit an alle $K$ üsten des Mittelmeeres wanderte und die Grundlage der herrschenden $\mathrm{Ge}$ wichts- und Münzsysteme des Alterthums bildete ${ }^{2}$ ), ist im alten Orient sehr frühzeitig in Geltung gewesen und hat in der alten babylonischen Doppelwährung und in verschiedenen abgeleiteten Systemen den Weltmarkt beherrscht.

In Aegypten galt ursprünglich ein anderes, von dem babylonischen verschiedenes Gewicht; aber dass die Tribute asiatischer Städte und Fürsten an die Aegypterkönige, so an Thutmosis III. ursprünglich nach baby-

1) Burraburias I Rückseite Z. $18 \mathrm{ff}$.

2) Dies ist von BöckH und BRANDIS, wenn auch nicht in so ausgedehntem Masse, vermuthet und erkannt, aber nur theilweise erwiesen worden. Den ausführlichen Beweis, dass die herrschenden Gewichtssysteme des Alterthums aus dem altbabylonischen System in einer Weise hergeleitet sind, dass sie in ihren Namen die organische Zugehörigkeit zu diesem System mit überraschender Genauigkeit gewahrt haben, werde ich demnächst an anderem Orte zu erbringen suchen. S. einstweilen: Archaeologische Gesellschaft zu Berlin. Sitzungsberichte, November 1888. 
lonischem Gewicht normirt waren, hat man seit langem erkannt ${ }^{2}$.

In diesem Sinne ist z. B. die Gewichtsberechnung des von Burraburias an Amenothes gesandten kostbaren Gesteins nach Mine ${ }^{2}$ ), sowie die Angabe des von dem Babylonier erbetenen Goldes in Talenten ${ }^{3}$ ). Angaben, welche Bekanntschaft oder Bekanntwerden der Aegypter mit diesem Gewichte erkennen lassen, von besonderem Interesse.

Die in dieser flüchtigen Uebersicht angedeutete Mannigfaltigkeit der Richtungen, nach denen der neue Fund unsere Kenntnisse bereichern kann, erfordert eine vielseitige Bearbeitung und Mitwirkung und rechtfertigt unsere Absicht, wenigstens einen kleinen Theil desselben der weiteren Forschung zugänglich $z u$ machen.

Ueber die Art der Edition und der Erklärung schicke ich noch wenige Worte voraus:

Die von mir angefertigten Autographien, bei denen ich mich bemühe deutlich zu sein und die Formen des Originals möglichst genau wiederzugeben, werden hoffentlich genügen, um ein vorläufiges Bild von dem Schrifttypus der betreffenden Documente $z \mathrm{u}$ geben.

Von den Texten gebe ich die Transscription, aus der für den Kundigen bereits hervorgeht, wie der Text im Wesentlichen verstanden ist. A uf eine vollständige Uebersetzung werde ich meist verzichten, weniger wegen der lexicalischen Schwierigkeiten, obschon auch diese nicht gering anzuschlagen sind, als wegen der Unklarheiten der Syntax. Wer sich vor Augen hält, wie selbst in den neueren Uebersetzungen gewöhnlicher assyrischer historischer Texte die Unkenntnis und Missachtung der Syntax das Verständnis der Texte beeinträchtigt, wie schöngefügte Perioden, auf deren mühsam

1) BRANDis, S. $91 \mathrm{ff}$.

2) Burraburias I, Rücks. Z. Io.

3) Burraburzas 3, Rücks. vorletzte Zeile. 
gelungenen $\mathrm{Bau}$ der assyrische Verfasser mit gerechtem Stolze blicken mochte, vom Uebersetzer unbarmherzig in coordinirte Sätzchen zerrissen werden, dem wird es klar sein, warum ich die Syntax als die dunkelste Seite dieser Texte betrachte, welche ohnehin der Aufhellung so grosse Schwierigkeiten entgegensetzen.

Ich werde im Commentar diejenigen Sätze, die vollständig erhalten und in ihrem Bau klar sind, im Zusammenhang geben und übersetzen. Im Uebrigen bitte ich zu beachten, dass es vorläufige Beiträge zur Erklärung sind, die gegeben werden sollen, und keine Sprache und Inhalt des Fundes erschöpfenden Abhandlungen, die erst in viel späterer Zeit geschrieben werden können. Auf Combinationen lasse ich mich nicht ein und werde in manchen Punkten ein Nichswissen bekennen, wo ich eine Vermuthung an die Stelle setzen könnte. Dass ich zur sprachlichen und inhaltlichen Erklärung nur clie in Berlin vorhandenen Tafeln heranziehen kann, von den in L, ondon und in Bulaq aufbewahrten nur, was inzwischen publicirt werden sollte, hebe ich ausdrücklich hervor.

I. Zur Correspondenz des Königs Burraburiaš mit Amenothes IIII von Aegypten.

Das wichtigste und umfassendste Schriftstück dieser Gruppe, das zugleich den Schlüssel zum Verständnis des ganzen Fundes geliefert hat (s.o. S. 373 f.), ist der passend wohl mit Burraburiaš I $z u$ bezeichnende Brief, auf einer Tafel aus chocoladebraunem Thon sehr heller Färbung. H. o, r65, B. ca. o, $08 \mathrm{~m}$. Sehr schön und deutlich geschrieben. Langte leider mit zerbröckelten Rändern in Berlin an, sodass Anfang und Ende der Zeilen vielfach gelitten haben. Herausgegeben nach dem Original, einer Photographie und meiner Copie vom 5/III 88. 


\section{Transscription.}

Vorderseite.

I. [Ana Na-ap-bú]-ru-ri-ia sarri rabî sarri mâti Mi-iș[-ri]

2. [ki-bi]-ma um-ma: Bur-ra-bu-ri-ia-[aś]

3. [sarru rabû sar] mâti Ka-ra-ilu-Du-ni-ia-aš a-h[u-ka]

+. [A-na ia]-a-sí u bîti-ia a-na sisê-ia u [narkabâti]-ia

5. [a-na r]a-ab-bu-ti-ia u ma-ti-ia da-an-ni[-iš lû sul-mu].

6. A-na a-bi-ia u bîti-su a-na sisê-su u [narkabâti-su].

7. a-na ra-ab-bu-ti-su u ma-ti-su da-an-ni-iš l[u sul-mu].

8. Ul-tu û(m)-mi ša mâr ši-ip-ri ša a-bi-ia ik-

9. [š-ip-]ri ul ța-ba-an-ni-ma mâr si-ip-ri-šu ai

10. ...... ni-ia a-ka-la ul i-ku-ul u si-ka-ra

II. . . . . u mâr sip-ri-ka ta-sa-'-al ma i-g a-

12. . . . . [si-]ip(?)-ri la ța-ba-an-ni-ma a-na na-

13. . . . . . . ma-ma la ta-du . . . .

14. . . . . . i i]p(?)-ri la ta-ba-an-ni-ma a-bu-u-a ri-e-s[i?]

15. a-na-ku ......-li(?)- ib-ba-ti iş(???)-bi(?) sa a-hi-ia am...

16. um-ma-a: ki-i ma-ar-șa-ku a-bu-u-a ul iš-

17. am-mi-ni ri-e-si la iš .....

18. mâr si-jp-ri-šu am-mi-ni la iš-pu-ra-am-ma la i-mu-[ru]

19. nıâr sii-ip-ri sa a-bi-ia an-ni-ta ik-ta-ba-a

20. ..... ....-a: ul ga-ag-ga-ru ki-ir(??)-bu-um-ma

21. a-bu-ka i-se-im-me-ma šu-ul-ma i-sa-ap-pa-ra-ak-ku

22. ma-tum ru-ga-at a-na a-bi-ka ma-an-nu(?) i-gal-ba-assu-um-ma

23. su-ul-ma ba-mu-ut-ta i-ša-ap-pa-ra-ak-ku,

24. ki-i ma-ar-șa-ta-a a-ḩu-ka i-še-im-me-e-ma

25. u mâr ši-ip-ri-šu ul i-ša-ap-pa-ra-ak-ku

26. a-na-ku a-ka-an-na ak-ta-ba-aś-su um-ma-a a-na a-bi-ja:

27. šarru rabâ, ma-tum ru-uk-tu-u i-ba-aš-ši u ki-ru-ubtum i. ba-ass-si.

28. Šu-u a-ka-an-na ik-ta-ba-a um-ma-a: Mâr ši-ip-ri-ka sa(??)-a-al

29. ki-i ma-tum ru-ga-tu-ma ina su-mi-ka a-bu-ka la išmu-u-ma 
30. a-na su-ul-mi-ka la iš-pu-ra

31. i-na an-na ki-i mâr si-ip-ri-ia a-sa-lu-ma ik-ba-a

32. ki-i gi-ir-ru ru-ga-a-tum li-ib-ba-at a-hi-ia ul am-lakan(?)-ni

33. u ki-i ik-bu-ni: i-na ma-ti sa a-bi-ia

34. ga-ab-bu i-ba-as-si u a-bu-u-a mi-im-ma-ma ul ha-si-ih

35. u i-na ma-ti-ia ga-bu-um-ma i-ba-aš-si .....

.j6. u a-na-ku mi-im-ma-ma ul ha-as-ba-[ku],

37. a-ma-ta ba-ni-ta sa ul-tu pa-na i-na ga-at sar-ra-ni...

38. ma-bi-ra-nu-ma su-ul-ma a-na a-ha-mi iš ni-sa-ap-pa[-ra-ak]

39. ši-i-ma a-ma-tum i-na bi-ri-ni lu ka-ai-na.

40. ... . . i a-na ........... .

(Unterer Rand abgebröckelt.)

Rückseite.

(Zu Anfang fehlen mindestens 3 Zeilen.)

I.

2. su-ul(?) ........ u su-lum-ka i-. . .

3. at-ta i-na ........ ri id-du-u si-it-ta (?) . . . .

4. ma-ar si-ip-ri-ia ta-ak-ta .....

5. mâr si-ip-ri-ka ți-e-ma al-ta-ka-an ma al-ta-kan(?)...

6. mâr si-ip-ri-ia ba-mu-ut-ta ți-e-ma su-ku-un-ma li (?) . . .

7. u ki-i ik-bu-ni-im-ma gi-ir-ru da-an-.....

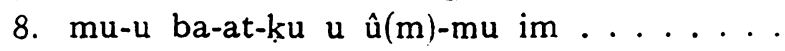

9. su-ul-ma-na ma-'a-da ba-na-a ul u-se-bi-la ak ....

Io. 4 ma-na abni Za-gin ba-na-a ki-i su-ul-ma-an ga-ti...

II. a-na a-bi-ia ul-te-bi-la

12. u 5 și-mi-it-ta sa si-si-i a-na a-bi-ia ul-te-bi-la

13. ki-i $\mathfrak{a}(\mathrm{m})-\mathrm{mu}^{\mathrm{x}}$ ) iț-ți-bu (?) mâr si-ip-ri-ia ar-ku-u sa il-la-ka

14. su-ul-ma-na ba-na-a ma-'a-da-a-na a-bi-ia u-se-ib-bi-la 15. u mi-nu-u sa a-bu-u-a ba-aš-bu a-bu-u-a li(?)-iš-pu-raam-ma

1) Oder (?) ki i-tam-ma. 
16. ul-tu bi-ti-su-nu li-il-ku-ni-iš-su

17. du-ul-la șa-ab-ta-ku-ma a-na a-bi-ia as-pu-ra

18. a-bu-u-a burâșa ba-na-a ma-'a-da li-se-bi-la-am-ma

ig. a-na du-ul-li-ia lu-us-ku-un

20. u hurâșu sa a-ḅu-u-a u-se-ib-bi-la

21. [a]-hu-u-a a-na pa-an ga-aia (?)-ni ma-am-ma la u-masa-ar.

22. ... a-hi-ia li-mu-ra-ma a-hu-u-a li-ik-nu-uk-ma li-se-bi-la

23. . . . ma-hi-ra-a sa a-bu-u-a u-se-bi-la ki sá a-huu-a ul i-mu-ur

24. ...... a(?) pa-nu-um-ma sa a-hi-ia ik-nu-uk-ma li-se-bi-la .

25. .... na burâșu sa na-šu-ni a-na u-tu-ni ki-i aș ?... 26. . . . . . . na(?)-ar-ru-um-ma ul i-la .... .

27. ... m. Șa-al-mu mâr si-ip-ri-ia sa asł-pu-ra-ak .... 28. . . . ni-su gi-ir-ra-su ha-ab-[ta ..... . ]

29. . . . . te te-it m. Bi-ri-ia-ma-za ih-ta-ba

30. ....... ni-ta gi-ir-ra-su m. Pa-ma-hu

31. ...... se-ir ma-ti-ka sa ma-at ki-iṣ-ri ih-ta-ba ...

32. ... di-na sa-a-šu a-ḩu-u-a ....

33. ...... mâr si-ip-ri-ia a-na pa-an a-bi-ia it $\ldots \ldots$ 34. ...... an-na m. Sa-al-mu a-na pa-an a-hi-ia it ... 35. ..... e-šu li-te-ir-ru-ni ........ 36. .... hi-bi-il-ta-šu li-sa-al-li-mu.

\section{Commentar.}

Aus dem an sich nicht einfachen Texte, dessen Verständnis noch besonders durch die Verstümmelung der Vorderseite bedauerlich erschwert wird, heben sich die folgenden Abschnitte mit ziemlicher Deutlichkeit heraus:

I) Eingangsformel (Vorderseite Z. $1-7$ );

2) Klage des Burraburias über eine Botschaft des Aegypterkönigs, die dem Babylonier Unerfreuliches gebracht hat; (Vorderseite Z. 8 ff.); 
3) Vorschlag, die Integrität des Gebietes und Besitzstandes sich gegenseitig $z u$ gewährleisten und frühere Bündnisse $z u$ erneuern (Vorderseite Z. 33-40);

4) Aufzählung der babylonischerseits gesandten Frieclensgeschenke und Ausbedingung der Gegenleistung (Rückseite Z. 5-26);

5) Klage über Beraubung (?) eines Boten cles Burraburias und Gesuch um Entschädigung (Rückseite Z. 2;-36).

[Vird fortgesetzt.] 


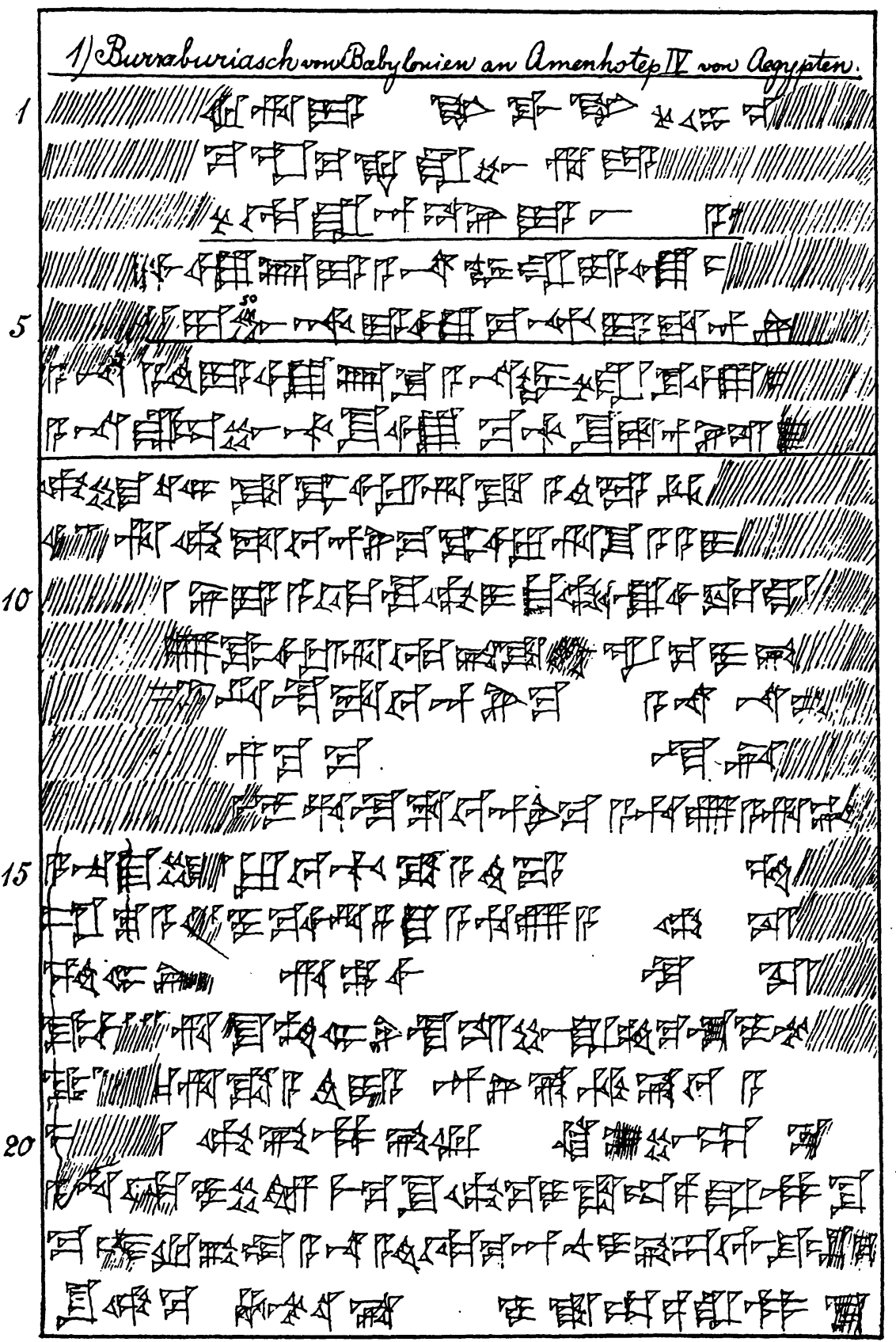




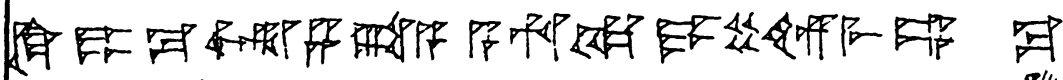

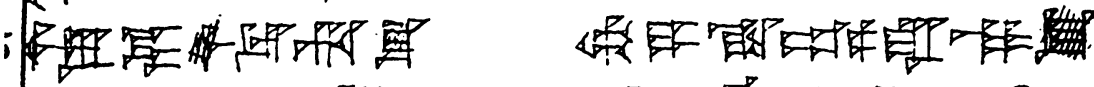

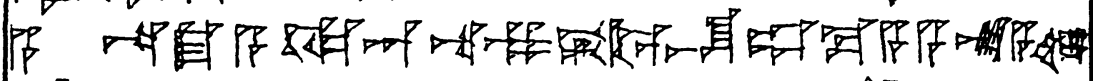

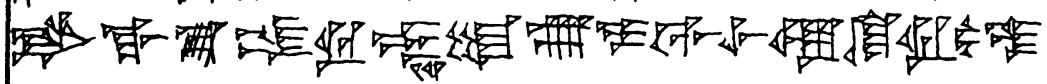

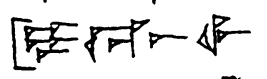

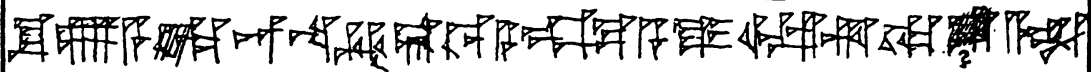

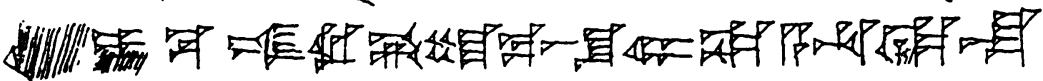

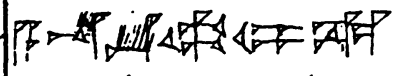

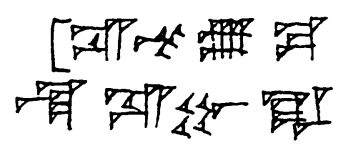

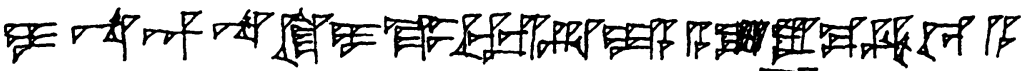

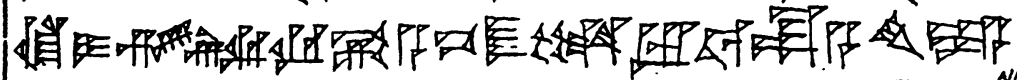

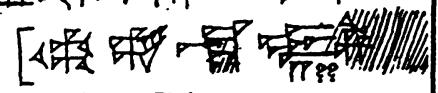

4

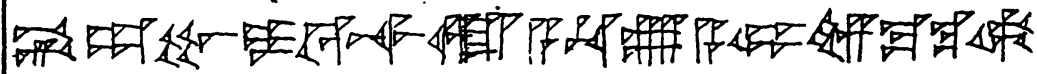

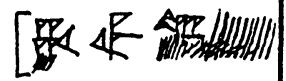

34

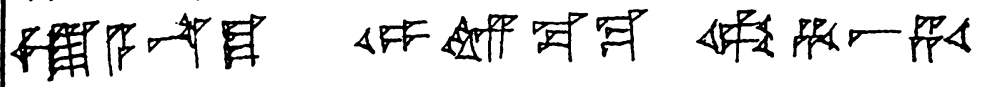

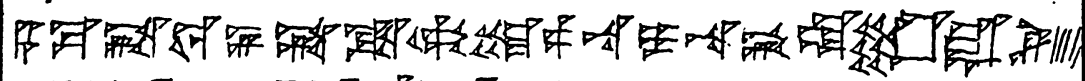

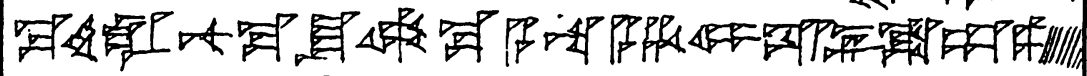

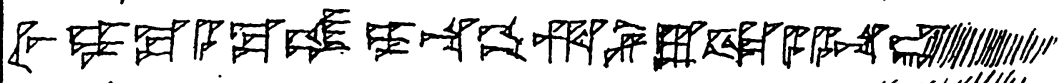

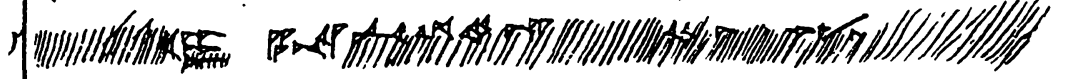
Qunterev Shand abgebrootien. 


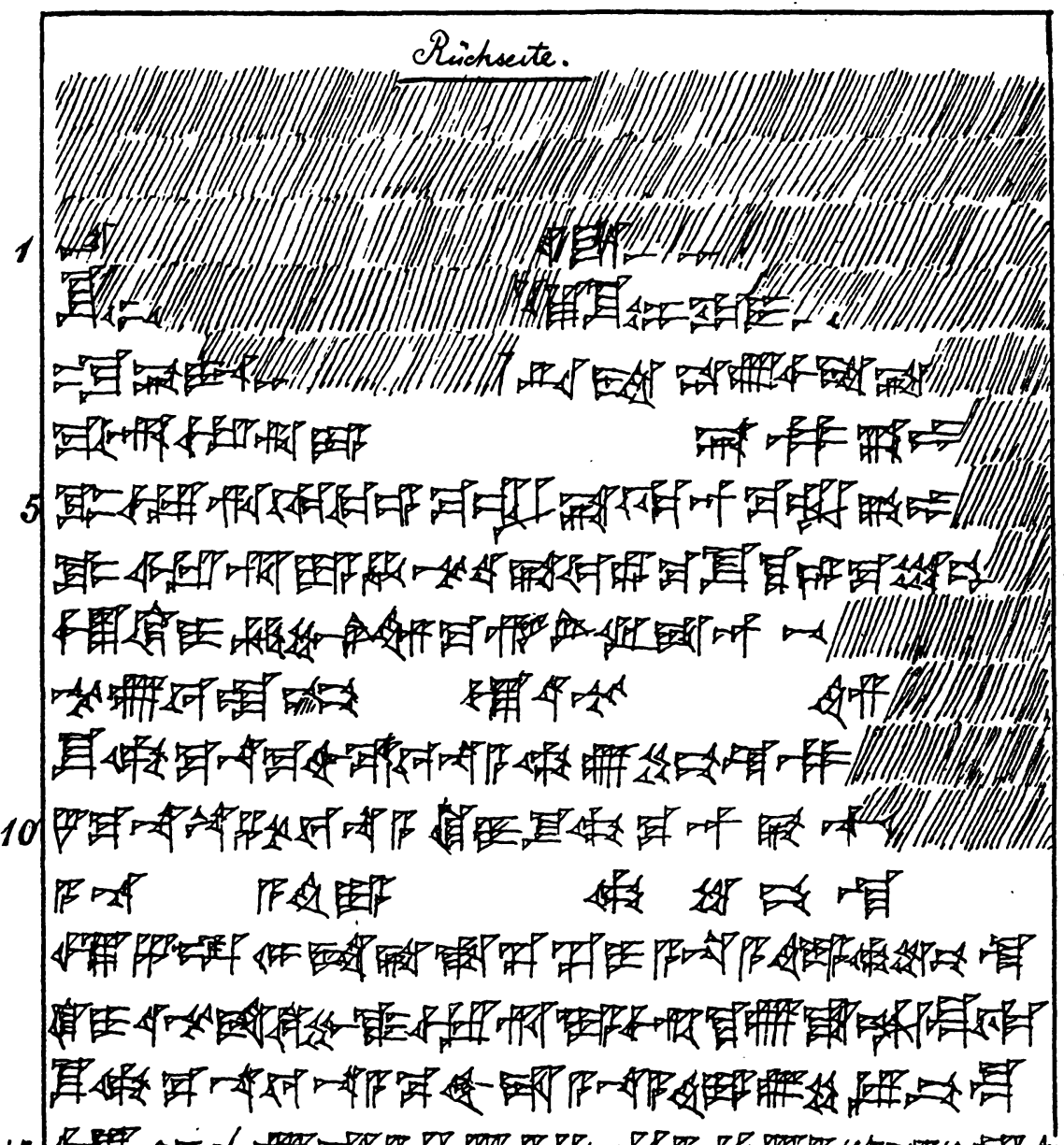

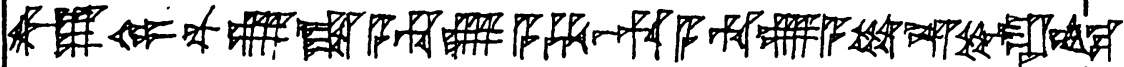

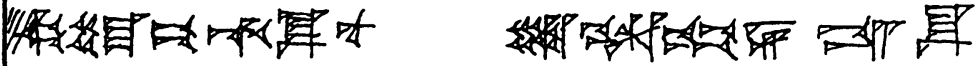

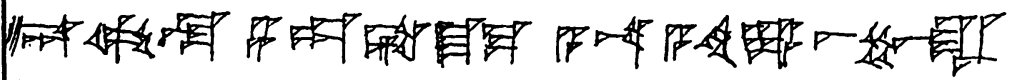

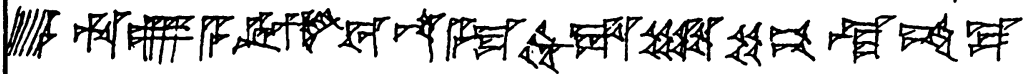

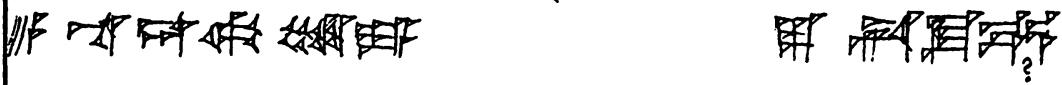
1) Gben mindestens 2 Zeilew dhebriohell und meggebrochen. 


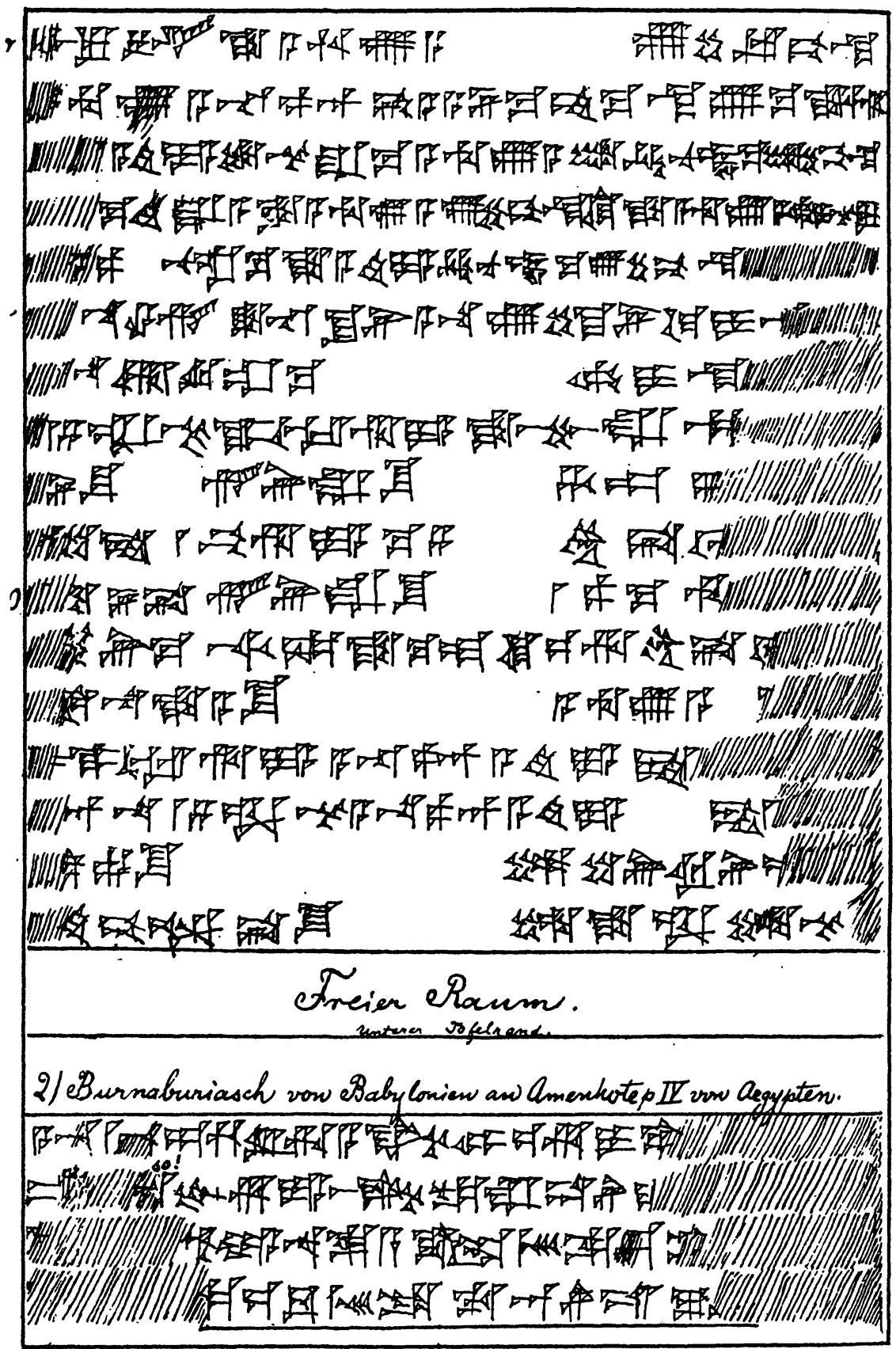


Aus dem Funde von Fell el Omarna.

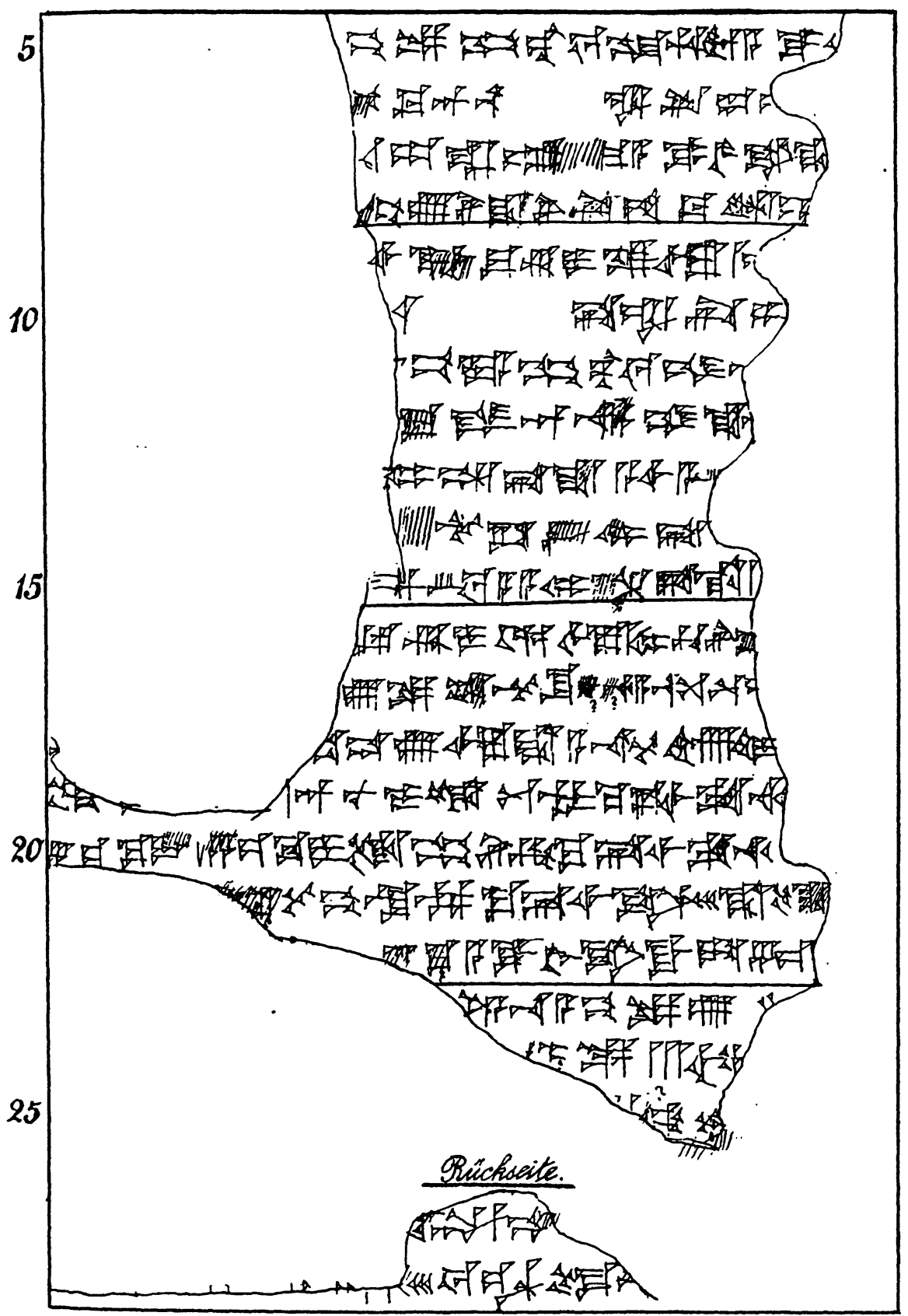




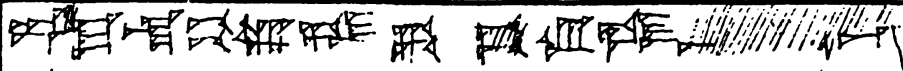

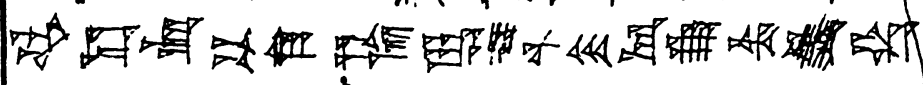

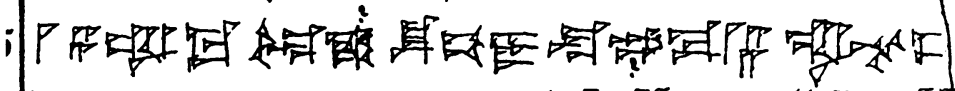

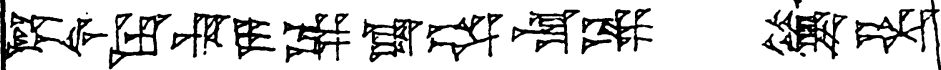

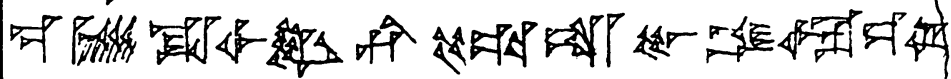

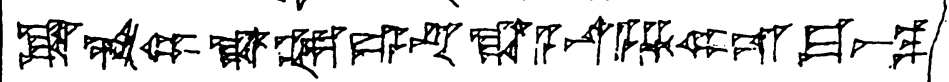

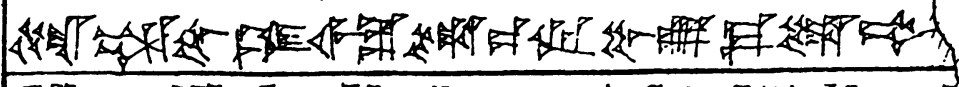

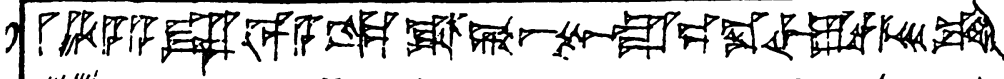

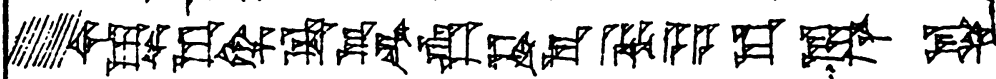

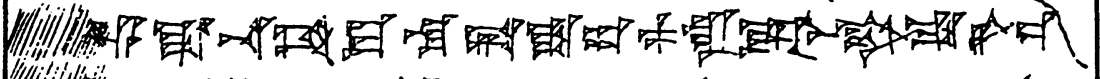

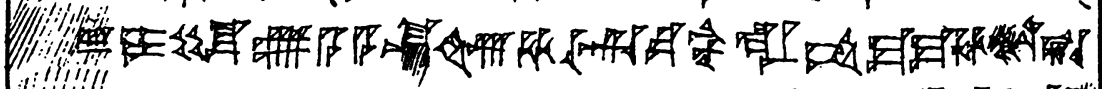

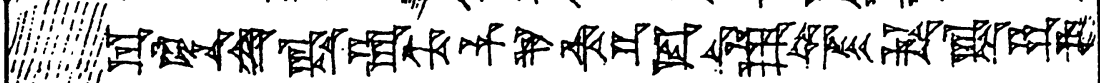

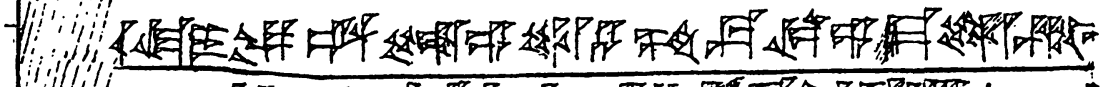

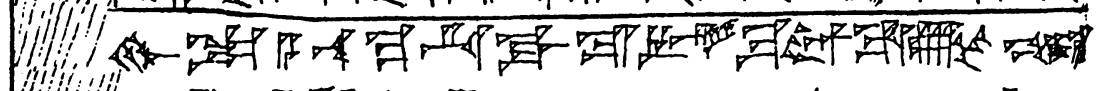

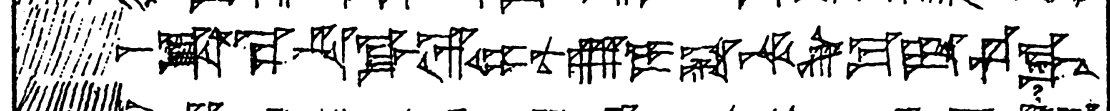

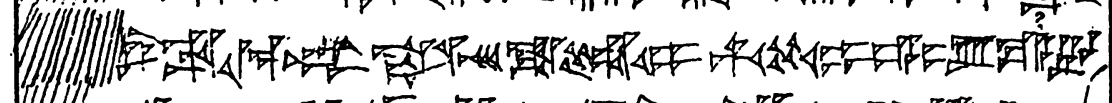

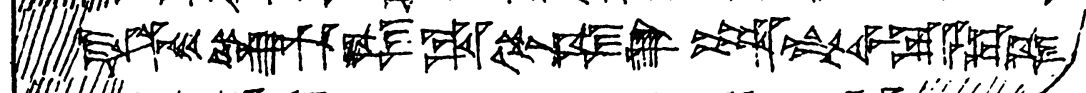

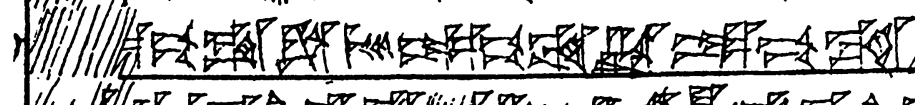

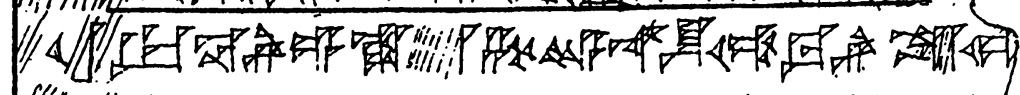

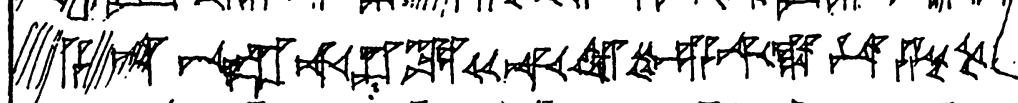

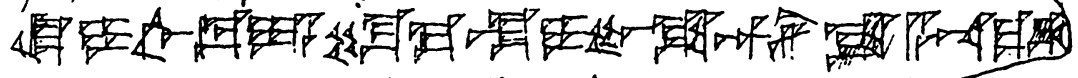

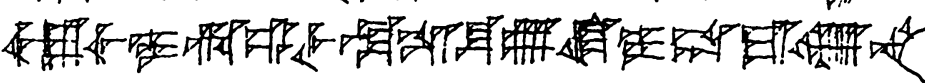

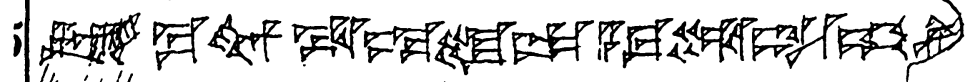

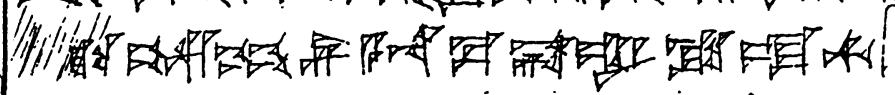




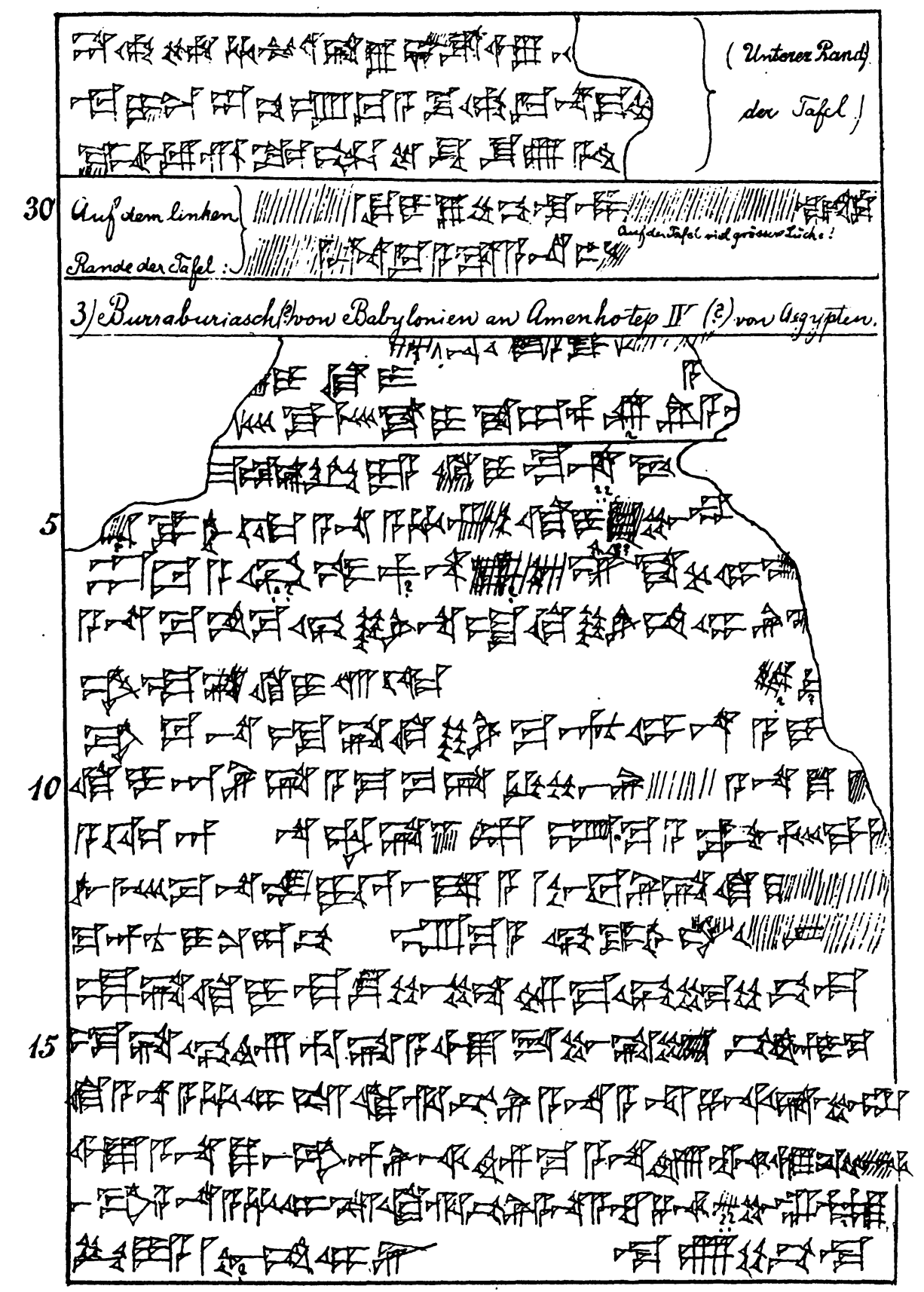


406 •. P. T. Sehmann, Elu dem Frunde von Fell el Elmarna.

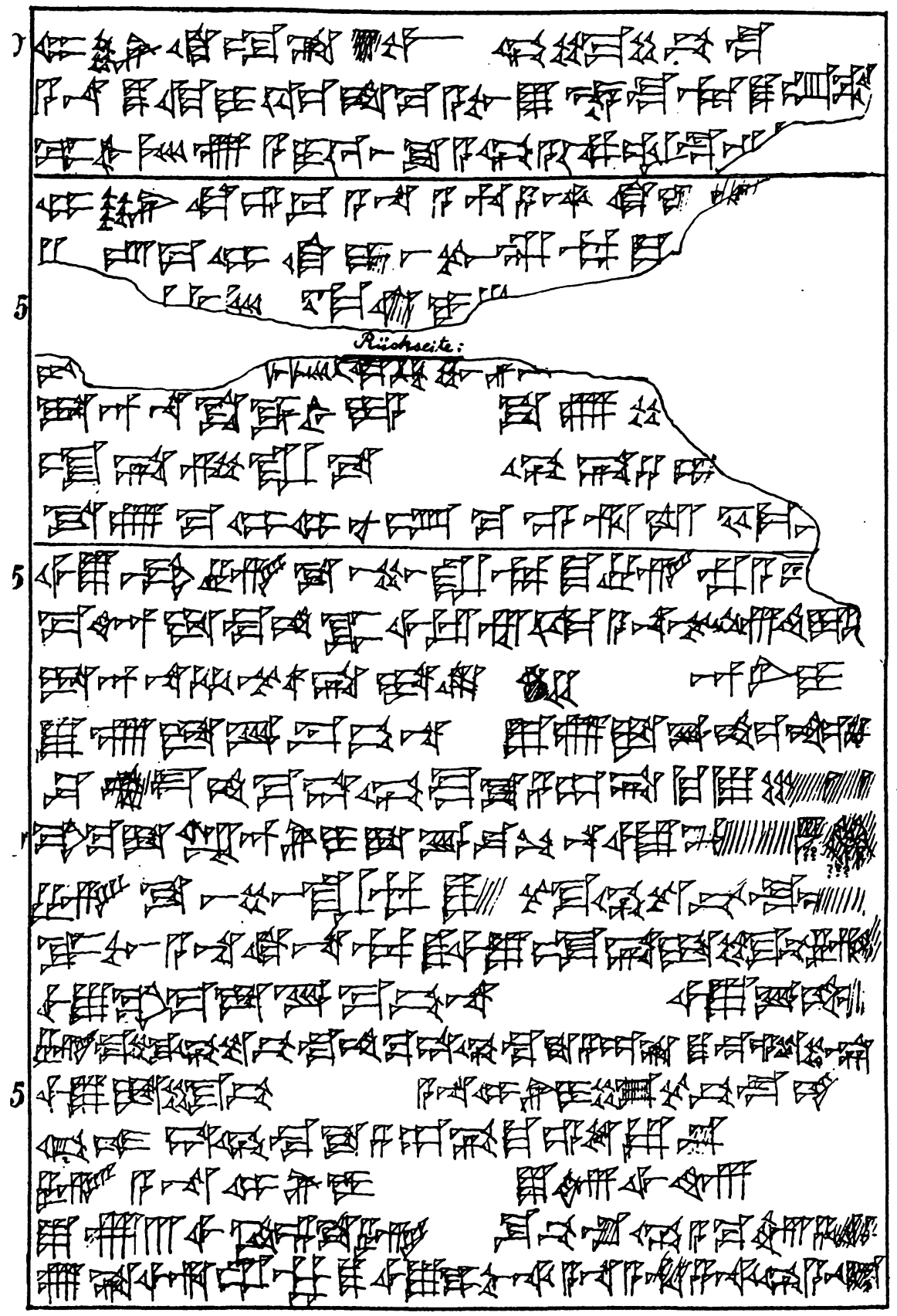

Weitere Taste folgens you by | Simion Fraser University 